\title{
Results of Gender-Specific Total Knee Arthroplasty: Comparative Study with Traditional Implant in Female Patients
}

\author{
Jong-Min Kim, $\mathrm{MD}^{1}$, Sang-Beom Kim, $\mathrm{MD}^{2}$, Jong-Min Kim, $\mathrm{MD}^{2}$, Dae-Hee Lee, $\mathrm{MD}^{3}$, Bum-Sik Lee, $\mathrm{MD}^{4}$, and \\ Seong-Il Bin, $\mathrm{MD}^{2}$ \\ ${ }^{1}$ Department of Orthopaedic Surgery, Kosin University Gospel Hospital, Busan; ${ }^{2}$ Department of Orthopaedic Surgery, Asan Medical Center, Seoul; ${ }^{3}$ Department of \\ Orthopaedic Surgery, Korea University Anam Hospital, Seoul; ${ }^{4}$ Department of Orthopaedic Surgery, Incheon St. Mary’s Hospital, Inchoen, Korea
}

Purpose: To compare the incidence of overhang between two distinct femoral components and whether there is clinical and radiological benefit of gender-specific implants in short-term follow-up.

Materials and Methods: One hundred and four knees in consecutive 66 female patients who underwent primary total knee arthroplasty due to primary osteoarthritis were included in this study. Overhang was measured and recorded in every cut surface of femur with both gender-specific and traditional trial femoral components respectively in every patient. Then, the knees were divided into two groups according to the type of the permanent femoral component they received. Clinical and radiological outcomes were compared between 2 groups at minimum 3 years after operation.

Results: Mean follow-up duration was 41.3 months (range, 36 to 50 months). Sixty two knees (59.6\%) showed femoral overhang at least in one area with a traditional trial component, while 26 knees $(25.0 \%)$ did with a gender-specific trial component ( $\mathrm{p}<0.001)$. In terms of range of motion, Hospital for Special Surgery knee score, radiographic result, patella tilt angle and displacement, no significant difference was observed between two groups. Conclusions: The use of gender-specific implants substantially reduced the incidence of femoral overhang but did not demonstrate any clinical, functional or radiologic benefit in short-term follow-up.

Keywords: Knee, Osteoarthritis, Arthroplasty, Gender, Female

\section{Introduction}

The history of total knee arthroplasty (TKA) designs demonstrates attempts at implants that more closely match patient anatomy for improved treatment results. Traditional total knee implant systems were designed to provide components of a wide range of sizes to achieve a proper fit in almost all patients.

Received January 15, 2014; Revised (1st) July 16, 2014;

(2nd) October 15, 2014; Accepted October 16, 2014

Correspondence to: Seong-Il Bin, MD

Department of Orthopaedic Surgery, Asan Medical Center, 88 Olympicro 43-gil, Songpa-gu, Seoul 138-736, Korea

Tel: +82-2-3010-3528, Fax: +82-2-488-7877

E-mail: sibin@amc.seoul.kr

This is an Open Access article distributed under the terms of the Creative Commons Attribution Non-Commercial License (http://creativecommons.org/licenses/by-nc/3.0/) which permits unrestricted non-commercial use, distribution, and reproduction in any medium, provided the original work is properly cited.
However, selection of a femoral component of correct size for a particular patient can still be challenging not only because it is common to have a distal femoral anteroposterior (AP) dimension going in the middle of usual 3-4 $\mathrm{mm}$ gap between each sizes of traditional femoral components but also because medial lateral (ML) overhang is sometimes encountered even with a traditional femoral component with a precisely matching AP dimension. In sizing of a femoral component, the AP dimension is important to maintain the flexion-extension gap and optimal tension in the extensor mechanism, whereas the ML dimension determines adequate coverage of the resected bone surface, allowing more even stress distribution and smooth tracking of the patellofemoral joint. Although there is no evidence on the clinical effect of underhang and overhang of implants, theoretical assumption is possible. If a component is too small (underhang), there will be higher contact stresses on the reduced bone-implant interface, increasing the risk of subsidence and loosening. Conversely, if a 
component is too large (overhang), it may impinge on the surrounding capsular tissues and ligaments, causing pain and limiting the range of motion (ROM) of the joint.

Anatomic variations between male and female are welldocumented in several anatomic and radiographic studies ${ }^{1-5)}$. In general, females tend to have smaller distal femora and different shapes with a narrower ML dimension for a given AP dimension when compared with males ${ }^{1}$. In addition, females are thought to have an increased quardriceps angle and a less prominent anterior femoral condyle than males even though controversy still remains ${ }^{6,7)}$.

The recent introduction of a gender-specific implant (Gender Solutions NexGen High-Flex; Zimmer Inc., Warsaw, IN) designed specifically for females started the debate that has focused on the effect of gender on the results of TKA using traditional implant systems. The Gender Solutions implant was designed to better accommodate the anatomic differences noted in females with a narrower ML dimension for any given AP dimension. Besides, the angle of trochlear groove was increased and the anterior flange thickness and width were reduced to more closely reproduce the native female anatomy.

With this background, we designed this present study in order to find out if gender-specific implants reduce the incidence of overhang during operation and there is any clinical or radiological advantage of gender specific implants over traditional implants. We hypothesized: 1) the incidence of femoral component overhang would be reduced with the use of gender-specific femoral components and 2) clinical and radiological results would show no difference between two types of implants in a shortterm follow-up because no clinical disadvantage of overhang has been reported so far.

\section{Materials and Methods}

\section{Patients}

Between May 2007 and January 2008, 136 primary TKAs were undertaken in a consecutive series of 92 patients. There were 7 males ( 8 knees) and 85 females (128 knees). The criteria for inclusion into the study consisted of female gender, a primary diagnosis of osteoarthritis and a minimum 3-year follow-up. Patients with severe bone loss requiring structural bone grafting or metal augmentation were excluded from the present study. Ultimately, 104 knees in 66 patients were included in the study. Their mean age at the time of the operation was 67.9 years (range, 52 to 80 years) and their mean body mass index (BMI) was $26.8 \mathrm{~kg} / \mathrm{m}^{2}$ (range, 20.2 to $33.3 \mathrm{~kg} / \mathrm{m}^{2}$ ). The mean follow-up duration was
41.3 months (range, 36 to 50 months). There were 28 unilateral procedures and 38 bilateral procedures that were performed two weeks apart. Of the 104 knees, only one had a valgus deformity. We compared the clinical and radiological outcomes of TKA using a gender-specific implant (Gender Solutions NexGen High-Flex) and a traditional implant (NexGen Legacy Posterior Stabilized Flex [LPS-Flex], Zimmer Inc.) at 3 years after surgery. We also analyzed intraoperative measurements, including the overhang of femoral component and patella tracking, after TKA using two different trial components.

\section{Surgical Technique}

All the operations were performed by the senior author (Bin) using the same technique. An anterior midline incision was followed by a medial parapatellar arthrotomy. After eversion of the patella, resection of the femoral condyles was performed to remove a thickness of bone equal to that of the femoral component to be inserted. The femoral cutting block was placed in $3^{\circ}$ to $5^{\circ}$ of external rotation to the posterior condyles, and this was ascertained by the AP trochlear sulcus. The valgus angle of femoral resection was made using an intramedullary guide, and AP cutting was performed using an anterior referencing system. Femoral component sizing was based on the AP dimension of the femur, which was measured with a femoral AP sizer during operation. If a measurement of the AP dimension fell between two sizes, the smaller size femoral component was chosen. Using an intramedullary tibial guide, a $10 \mathrm{~mm}$-thick tibial bone resection was performed to obtain a surface that was perpendicular to the shaft of the tibia in the coronal plane, with a $7^{\circ}$ posterior slope in the sagittal plane. Two different trial femoral components (Gender Solutions NexGen High-Flex prosthesis and NexGen LPS-Flex prosthesis) of the same size were inserted sequentially after femoral and tibial bone cuts. At this moment, overhang of each trial femoral component was examined medially and laterally at three cut surfaces (anterior, anterior chamfer and distal cut surfaces). Overhang was measured from the bone margin of each cut surface using a ruler. Overhang of more than $1 \mathrm{~mm}$ was considered to be positive. Thus, overhang was assessed and recorded at six areas in the distal femur. Patellar tracking was also checked with each trial component using the 'no thumb' test. The ROM in which the patella showed congruent tracking was recorded with each trial femoral component and designated as congruent patella tracking ROM. Based on the coverage of cut surface and patellar tracking, appropriate type of permanent femoral implant was chosen by the surgeon. The patients who received a gender-specific implant (Gender Solutions) were assigned to group I and those 
with a traditional implant (NexGen LPS-Flex) were to group II.

The rehabilitation program was the same in both groups. Immediately after surgery, patients were encouraged to begin quadriceps strengthening exercises. Two days after surgery, drain was removed and continuous passive motion and tolerable weight bearing were started. We also encouraged ROM and straight leg raising exercises. Patients were discharged from the hospital 10-12 days postoperatively.

\section{Evaluated Parameters}

The entire data were collected prospectively from the beginning of the present study and were compared and analyzed retrospectively. This study was approved by the Institutional Review Board at our hospital and informed consent was waived. Presence of overhang at the three femoral cut surfaces and the congruent patella tracking ROM of each prosthesis were assessed intraoperatively and compared between groups. The distribution of femoral component size in each group was also compared. Postoperatively, the patients were reviewed at six weeks, three months, six months, and annually after surgery. Clinical parameters including Hospital for Special Surgery (HSS) knee score ${ }^{8)}$, flexion contracture, maximal flexion, and complications were evaluated and compared preoperatively and at 3 years after surgery. Flexion contracture and maximal flexion were measured using a manual goniometer with the arms aligned along the long axes of the femur and tibia on the lateral side of the knee joint. Patients were told to straighten and bend their knee until they felt a slight degree of pain in supine position. The ROM was calculated by subtracting flexion contracture from maximal flexion. All the data at follow-up examinations were recorded by an orthopedic surgeon and compiled by a research assistant who was not a part of the surgical team and had no information about the surgical and radiological findings.

Radiographic evaluation included standing AP, lateral, and Merchant views of both knees at three months, six months, and annually after surgery, which were analyzed using the Radiological Evaluation System of the Knee Society ${ }^{9)}$ to delineate radiolucency around the components. Postoperative patellar tracking was assessed by measuring patella tilt angle and patella displacement as described by Gomes et al. ${ }^{10)}$. All the radiographs were analyzed by two of the co-authors (Kim JM and Kim SB) who were blinded to patient information.

All data were analyzed using SPSS ver. 12.0 (SPSS Inc., Chicago, IL, USA). The level of statistically significant difference was set at $\mathrm{p}=0.05$.

\section{Results}

The incidence of overhang of the trial femoral component was significantly higher in the knees with the traditional component than those with the gender-specific trial component (Table 1). Of the 104 knees, overhang was observed at least in one area in 62 knees with the traditional trial component (59.6\%) compared to 26 knees $(25.0 \%)$ with the gender-specific trial component $(p<0.001)$. Among the six areas where overhang was examined, the lateral anterior cut surface was the area where overhang of the femoral component was most frequent regardless of the type of the trial component. Distal medial area was the only exception that did not show statistically significant difference $(\mathrm{p}=0.250)$.

Sixty-two knees (59.6\%) that demonstrated overhang with traditional trial components were replaced with a gender-specific implant (group I) and the remaining 42 knees (40.4\%) that showed no overhang with traditional trial components were replaced with a traditional implant (group II). Therefore, the incidence of overhang after final implantation was 26/62 knees (41.9\%) in group I and no overhang (0/42 knees) was observed in group II. As a result, 36 knees in group I were able to avoid overhang with use of the gender-specific implants instead of the traditional ones, whereas the remaining 26 knees in group I still showed overhang even with gender-specific implants. Ten patients received a Gender Solutions prosthesis on one side and a LPS-Flex prosthesis on the other side. Those 10 patients were also included in the analysis of clinical and radiographic results. There were no demographic differences between the two groups (Table 2). The difference of the congruent patella tracking ROM

Table 1. Incidence of Overhang Using Each Trial Femoral Component $(\mathrm{n}=104)$

\begin{tabular}{lccc}
\hline $\begin{array}{c}\text { Area in distal } \\
\text { femoral cut surface }\end{array}$ & Gender-specific (\%) & Traditional (\%) & p-value ${ }^{\mathrm{a})}$ \\
\hline $\begin{array}{l}\text { Anterior } \\
\text { Medial }\end{array}$ & $7(6.7)$ & $28(26.9)$ & $<0.001$ \\
Lateral & $19(18.3)$ & $43(41.4)$ & $<0.001$ \\
Anterior chamfer & & & \\
Medial & $0(0)$ & $14(13.5)$ & $<0.001$ \\
Lateral & $4(3.9)$ & $33(31.7)$ & $<0.001$ \\
Distal & & & \\
Medial & $0(0)$ & $3(2.9)$ & 0.250 \\
Lateral & $4(3.9)$ & $28(26.9)$ & $<0.001$ \\
At least in one area & $26(25.0)$ & $62(59.6)$ & $<0.001$ \\
\hline
\end{tabular}

${ }^{\mathrm{a})} \mathrm{McNemar}$ test. 
Table 2. Patient Demographics

\begin{tabular}{lcclc}
\hline \multicolumn{1}{c}{ Variable } & Group I & Group II & Test & p-value \\
\hline No. of knees (patients) & $70(50)$ & $34(26)$ & Mann-Whitney test & 0.184 \\
Age $(\mathrm{yr})$ & $68.4 \pm 6.2$ & $67.0 \pm 6.8$ & Student t-test & 0.804 \\
Height $(\mathrm{cm})$ & $150.5 \pm 4.8$ & $150.7 \pm 4.7$ & Student t-test & 0.218 \\
Weight $(\mathrm{kg})$ & $60.0 \pm 7.5$ & $61.9 \pm 6.3$ & Student t-test & 0.240 \\
BMI $\left(\mathrm{kg} / \mathrm{m}^{2}\right)$ & $26.5 \pm 3.1$ & $27.3 \pm 2.6$ & Chi-square test & 0.331 \\
Right/left & $32 / 38$ & $19 / 15$ & Mann-Whitney test & 0.848 \\
Mechanical axis $\left({ }^{\circ}\right)$ & $9.9 \pm 5.4$ & $9.9 \pm 4.4$ & & \\
\hline
\end{tabular}

Values are presented as mean \pm standard deviation or number.

BMI: body mass index.

Table 3. Congruent Patella Tracking Range of Motion (ROM) with Each Trial Femoral Component Assessed by No Thumb Test ( $\mathrm{n}=104)$

\begin{tabular}{lccc}
\hline & Gender-specific & Traditional & p-value \\
\hline Congruent ROM $\left({ }^{\circ}\right)$ & $126.3 \pm 10.1$ & $128.5 \pm 3.6$ & 0.464 \\
\hline
\end{tabular}

Values are presented as mean \pm standard deviation.

${ }^{a)}$ Mann-Whitney test.

Table 4. Sizes of Selected Femoral Implants

\begin{tabular}{cccc}
\hline Size & Group I (\%) & Group II (\%) & p-value $^{\text {a) }}$ \\
\hline C & $5(8.1)$ & $11(26.2)$ & \\
D & $47(75.8)$ & $26(61.9)$ & \\
E & $10(16.1)$ & $5(11.9)$ & \\
Total & 62 & 42 & 0.045 \\
\hline
\end{tabular}

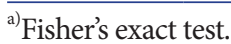

between traditional and gender-specific trial components was not significant $(p>0.05)$ (Table 3). Hence, there was no case where a gender-specific implant was chosen for better patella tracking. The distribution of the femoral component size ranged from $\mathrm{C}$ to $\mathrm{E}$ and showed a tendency of selecting larger sizes in the knees with the gender-specific implant ( $\mathrm{p}=0.045)$ (Table 4).

Clinical evaluation was performed in all patients including 10 patients who received different implants in bilateral TKA. The mean flexion contracture, mean maximal flexion and mean ROM improved significantly after operation in both groups. However, there was no significant difference between two groups. The mean HSS score also improved after operation in both groups and the difference between two groups was not significant (Table 5). Lateral release was not performed in both groups. At the final follow-up, the mean patella tilt angle and mean patella displacement showed no significant difference between two groups (Table 6). We observed no clinical complications in both groups, includ-
Table 5. Clinical Evaluations before Surgery and at Postoperative 3 Years

\begin{tabular}{lccc}
\hline \multicolumn{1}{c}{ Variable } & Group I & Group II & p-value $^{\text {a) }}$ \\
\hline Flexion contracture $\left(^{\circ}\right)$ & & & \\
Preop & $9.1 \pm 8.1$ & $8.2 \pm 6.4$ & 0.759 \\
Last F/U & $0.4 \pm 1.8$ & $0.3 \pm 1.7$ & 0.661 \\
p-value & & \\
Maximal flexion $\left(^{\circ}\right)$ & $<0.001$ & $<0.001$ & \\
Preop & & & \\
Last F/U & $126.6 \pm 14.1$ & $127.9 \pm 12.2$ & 0.856 \\
p-value & \\
ROM $\left({ }^{\circ}\right)$ & $130.6 \pm 11.0$ & $129.6 \pm 10.5$ & 0.522 \\
Preop & 0.031 & 0.042 & \\
Last F/U & & & \\
p-value & & & \\
HSS score & $117.6 \pm 18.4$ & $119.8 \pm 14.8$ & 0.832 \\
Preop & $130.2 \pm 11.8$ & $129.3 \pm 11.0$ & 0.563 \\
Last F/U $^{\text {b) }}$ & $<0.001$ & $<0.001$ & \\
p-value & & & \\
\hline
\end{tabular}

Values are presented as mean \pm standard deviation.

Preop: preoperative, F/U: follow-up, ROM: range of motion, HSS: Hospital for Special Surgery.

${ }^{a)}$ Mann-Whitney test. ${ }^{b}$ Wilcoxon signed rank test.

Table 6. Patella Tilt Angle and Displacement at Postoperative 3 Years

\begin{tabular}{lccc}
\hline \multicolumn{1}{c}{ Variable } & Group I & Group II & p-value ${ }^{\mathrm{a})}$ \\
\hline Patella tilt angle $\left(^{\circ}\right)$ & $6.0 \pm 3.1$ & $6.0 \pm 3.8$ & 0.949 \\
Displacement $(\mathrm{mm})$ & $-1.5 \pm 1.9$ & $-1.1 \pm 2.5$ & 0.606 \\
\hline
\end{tabular}

Values are presented as mean \pm standard deviation.

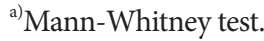

ing delayed wound healing, deep infection or instability. No indications of component loosening and progressive osteolysis were 
noted in any zone on the postoperative radiographs.

\section{Discussion}

In the present study, the incidence of overhang was reduced by $34.6 \%$ in the gender-specific implant group in which overhang was inevitable during trial insertion of the traditional implant. However, clinical disadvantage of overhang was not demonstrated in the short-term follow-up.

In 1996, Poilvache et al. ${ }^{4)}$ indicated some female femora were narrower than average and the available femoral component with adequate AP dimension might be too broad in these patients. Several years later, Chin et al. ${ }^{1)}$ investigated 200 consecutive knees (100 males and 100 females) during primary TKA and reported female femora showed a tendency to have a narrower ML dimension for any given AP dimension than male femora (AP/ML ratio, 0.82 vs. 0.79 ). Shortly after this study, Hitt et al. ${ }^{2)}$ performed a well-designed multicenter study enrolling 337 knees (128 males and 209 females) to compare six different contemporary prosthetic systems of traditional design. They reported that all the prostheses tended to present more or less ML overhang in female femora and the tendency was more evident in larger sizes. Moreover, they introduced the concept of aspect ratio (ML/AP ratio) and demonstrated that the aspect ratio of female femora tended to decrease in larger knees while male femora showed relatively constant aspect ratio throughout the sizes. After this study, the aspect ratio became a standard parameter to describe distal femoral morphology. They also stated manufacturers should consider gender-specific implants or decrease the ML dimension to prevent overhang in females. Stimulated by these reports, genderspecific implants were launched and marketed, which lead to the debate as to the relevance of this system ${ }^{6,7,11}$. Proponents of gender-specific implants presented accumulated data on sexual dimorphism of distal femur during decades and possible negative effects of component overhang ${ }^{6,11}$. On the contrary, opponents pointed out that most recent studies had failed to eliminate or correct for other biases between both sexes such as average height or size of the studied femora and concluded that the unproven use of gender-specific implants may pose ethical or medico-legal dilemmas ${ }^{7}$. In spite of this, there seems to be no disagreement at least on one point that the reported studies had neither directly demonstrated negative effects of overhang or overstuffing nor investigated actual merits of gender-specific knee arthroplasty with real implants.

The paucity of data on negative effects of overstuffing has been taken for granted due to the difficulty of measuring the amount of overhang and controlling multiple possible prognostic factors other than overhang. However, no theoretical advantage was expected with regard to the overhang of femoral implant. Thus, we decided to investigate the reduced incidence of overhang in TKA using gender-specific implants in Asian female patients even though the practical advantage of reduced overhang remained unclear. In this study, the incidence of ML overhang after TKA using traditional and gender-specific trial components showed a significant difference (59.6\% vs. $25.0 \%$ ). In other words, $34.6 \%$ of the patients were able to avoid femoral component overhang because gender-specific implants were available. Regarding the incidence of $25.0 \%$ of overhang even with the gender-specific trial component, these patients would have had greater overhang if it had not been for a gender-specific implant. With respect to the incidence of overhang in six separate areas, only distal medial cut surface did not show significant differences between two groups. This may be attributable to our efforts to lateralize femoral components during operation in order to improve patella tracking regardless of the type of implant. The distribution of selected sizes in each group showed a tendency of higher selection rate of a gender-specific implant in larger sizes $(\mathrm{p}=0.045)$. Our data is consistent with those of Hitt et al. ${ }^{2)}$ that showed a higher aspect ratio in smaller knees and a proportionally lower ratio in larger knees.

Recently, a few short-term clinical and radiologic outcomes were reported. Song et al. ${ }^{12)}$ compared clinical and radiologic results of TKA using gender-specific implants and conventional unisex design with a minimum follow-up of two years. They found no difference in clinical and radiologic outcomes between two groups and concluded gender-specific implants demonstrated no advantage over standard unisex design. Guy et al. ${ }^{13)}$ compared 50 males and 50 females to investigate the difference in the morphology of distal femur and the incidence of femoral component overhang. The mean aspect ratio was larger in females (1.02) than males $(0.98)(\mathrm{p}=0.005)$. Standard implants in TKA resulted in a significant increase of overhang in females in terms of both incidence and magnitude than did gender-specific implants. Tanavalee et al. ${ }^{14)}$ performed a prospective study that compared standard and gender-specific implants with an average followup of two years. Clinical and radiologic assessment revealed no difference between two groups. The selection rate of genderspecific implants was significantly higher in females (60.8\%) than males (8.2\%) and significantly increased with increasing femoral size. The latter finding was exactly concordant with that in our present study and the selection rate of gender-specific implants in females (60.8\%) was very similar to that of our study (59.6\%). 
Clarke and Hentz ${ }^{15)}$ reported radiographic results of TKA using gender-specific implants. They evaluated 122 knees in three subdivided groups (42 females with unisex implants, 41 males with unisex implants and 39 females with gender-specific implants) and concluded that traditional unisex femoral components did not accurately match female anatomy. However, they were not able to demonstrate better radiographic results with gender-specific implants. Therefore, the overall conclusion of the study was similar to ours in that traditional femoral components did not seem pertinent to reproduce female anatomy and gender-specific components did not provide a perfect solution, either.

Asymmetrical component sizes are known to be frequently used in bilateral TKA, and the prevalence of which ranges from $6.7 \%$ by Brown et al. ${ }^{16)}$ to $31 \%$ by Hitt et al. ${ }^{2)}$. In our study, 10 patients had a Gender Solutions prosthesis in one knee and a LPS-Flex prosthesis in the contralateral knee. This accounted for 10/38 $(26.3 \%)$ of all bilateral surgeries in the present study. Not only the size but also the shape of both distal femora of a patient may be different from each other according to our data, which alerted us to the risk of the use of the same implant in bilateral TKA. We believe the phenomenon of asymmetric component implantation probably originated from the awareness of the fact that anatomy is different from one side to the other.

The clinical outcomes of two groups did not present significant differences in terms of motion, performance of patella and HSS score in this study. Because these parameters are not considered to deteriorate rapidly in short term, long-term observation seems to be necessary to elucidate differences, if any, in clinical outcomes between two groups.

One of the limitations of this study is that it is not a true comparative study: implant selection relied on the extent of overhang of each trial femoral component and therefore the true independent clinical outcomes of each implant system were not thoroughly investigated. Besides, the minimum 3-year follow-up period may be considered insufficient to determine the longevity of clinical outcomes. However, this study is based on solid evidence from prospective data, which can be regarded as a strength of the study.

In the present study, the gender-specific femoral component explicitly reduced the incidence of overhang compared to the traditional femoral component. However, we could not find any correlation between this apparent advantage and more favorable clinical and radiologic outcomes. Potential advantages of gender specific implants, such as avoidance of overstuffing in the patellofemoral joint, soft tissue irritation and anterior knee pain, were not identified in this study.

\section{Conclusions}

There was significant difference in the incidence of overhang of femoral component between the gender-specific implant and the traditional implant. It was observed that $34.6 \%$ of the patients were able to avoid femoral component overhang with genderspecific implants. After three years of follow-up, there was no significant difference in clinical and radiological outcomes between two groups. Potential advantages of gender specific implants, such as avoidance of overstuffing in the patellofemoral joint, soft tissue irritation and anterior knee pain, should be evaluated in a long-term follow-up study.

\section{Conflict of Interest}

No potential conflict of interest relevant to this article was reported.

\section{Acknowledgments}

This research was supported by Basic Science Research Program through the National Research Foundation of Korea (NRF) funded by the Ministry of Education, Science and Technology (2012-0000479).

\section{References}

1. Chin KR, Dalury DF, Zurakowski D, Scott RD. Intraoperative measurements of male and female distal femurs during primary total knee arthroplasty. J Knee Surg. 2002;15:213-7.

2. Hitt K, Shurman JR 2nd, Greene K, McCarthy J, Moskal J, Hoeman T, Mont MA. Anthropometric measurements of the human knee: correlation to the sizing of current knee arthroplasty systems. J Bone Joint Surg Am. 2003;85 Suppl 4: 115-22.

3. Kwak DS, Surendran S, Pengatteeri YH, Park SE, Choi KN, Gopinathan P, Han SH, Han CW. Morphometry of the proximal tibia to design the tibial component of total knee arthroplasty for the Korean population. Knee. 2007;14:295300.

4. Poilvache PL, Insall JN, Scuderi GR, Font-Rodriguez DE. Rotational landmarks and sizing of the distal femur in total knee arthroplasty. Clin Orthop Relat Res. 1996;(331):35-46.

5. Vaidya SV, Ranawat CS, Aroojis A, Laud NS. Anthropometric measurements to design total knee prostheses for the Indian population. J Arthroplasty. 2000;15:79-85. 
6. Conley S, Rosenberg A, Crowninshield R. The female knee: anatomic variations. J Am Acad Orthop Surg. 2007;15 Suppl 1:S31-6.

7. Merchant AC, Arendt EA, Dye SF, Fredericson M, Grelsamer RP, Leadbetter WB, Post WR, Teitge RA. The female knee: anatomic variations and the female-specific total knee design. Clin Orthop Relat Res. 2008;466:3059-65.

8. Insall JN, Ranawat CS, Aglietti P, Shine J. A comparison of four models of total knee-replacement prostheses. J Bone Joint Surg Am. 1976;58:754-65.

9. Ewald FC. The Knee Society total knee arthroplasty roentgenographic evaluation and scoring system. Clin Orthop Relat Res. 1989;(248):9-12.

10. Gomes LS, Bechtold JE, Gustilo RB. Patellar prosthesis positioning in total knee arthroplasty: a roentgenographic study. Clin Orthop Relat Res. 1988;(236):72-81.

11. Greene KA. Gender-specific design in total knee arthroplasty. J Arthroplasty. 2007;22(7 Suppl 3):27-31.
12. Song EK, Jung WB, Yoon TR, Park KS, Seo HY, Seon JK. Comparison of outcomes after bilateral simultaneous total knee arthroplasty using gender-specific and unisex knees. J Arthroplasty. 2012;27:226-31.

13. Guy SP, Farndon MA, Sidhom S, Al-Lami M, Bennett C, London NJ. Gender differences in distal femoral morphology and the role of gender specific implants in total knee replacement: a prospective clinical study. Knee. 2012;19:2831.

14. Tanavalee A, Rojpornpradit T, Khumrak S, Ngarmukos S. The early results of gender-specific total knee arthroplasty in Thai patients. Knee. 2011;18:483-7.

15. Clarke HD, Hentz JG. Restoration of femoral anatomy in TKA with unisex and gender-specific components. Clin Orthop Relat Res. 2008;466:2711-6.

16. Brown TE, Diduch DR, Moskal JT. Component size asymmetry in bilateral total knee arthroplasty. Am J Knee Surg. 2001;14:81-4. 